\title{
A strongly non-Ramsey uncountable graph
}

by

\author{
Péter Komjáth (Budapest)
}

\begin{abstract}
It is consistent that there exists a graph $X$ of cardinality $\aleph_{1}$ such that every graph has an edge coloring with $\aleph_{1}$ colors in which the induced copies of $X$ (if there are any) are totally multicolored (get all possible colors).
\end{abstract}

An important chapter of combinatorial set theory is partition calculus which concerns the infinite generalizations of Ramsey's theorem. One of its corner stones is the Erdős-Rado theorem which says that if $\kappa, \mu$ are arbitrary cardinals then the complete graph on a large enough set has the property that whenever the edges are colored with $\mu$ colors then there is a monocolored subset of cardinal $\kappa$ (see Chapter 17 of [1]). For $\kappa=\aleph_{1}$, $\mu=\aleph_{0}$ the underlying set must have cardinality $\left(2^{\aleph_{0}}\right)^{+}$and this is sharp. If the cardinal of the ground set is not enough large then - as discovered by Erdős, Hajnal, and Rado - very strong counterexamples exist, examples of $\mu$-colorings, in which every $\kappa$-subset of the underlying set has edges in every color. An important example in this direction is the Todorčević coloring, i.e., a function $h:\left[\omega_{1}\right]^{2} \rightarrow \omega_{1}$ with the property that if $X \subseteq \omega_{1}$ is uncountable then $h$ assumes every value on $[X]^{2}$ (see [4]). This is usually denoted by $\omega_{1} \nrightarrow\left[\omega_{1}\right]_{\omega_{1}}^{2}$.

Another direction in Ramsey theory is when we want not necessarily complete monochromatic graphs but to exclude trivialities we require that the required monocolored copy of the predetermined graph be induced. If the graph and the number of colors are both finite then there always exists a graph with the required property. It was shown, however, by A. Hajnal and the author that at least consistently there may be a negative answer. In [2] we proved that if a Cohen real is added to a model of set theory then in the resulting model there is a graph $X$ such that every graph has

1991 Mathematics Subject Classification: 05D10, 03E35, 05C55.

Partially supported by the European Communities (Cooperation in Science and Technology with Central and Eastern European Countries) contract no. ERBCIPACT930113. 
an edge-coloring with two colors and with no monochromatic induced copy of $X$. Soon after S. Shelah proved the consistency of the other direction, that for any graph $X$ and any cardinal $\mu$ there is a graph which always has monocolored copies of $X$ when its edges are $\mu$-colored ([3]).

In this note we show that there may consistently exist a graph with a very strong non-Ramsey property, a graph $X$ of cardinal $\aleph_{1}$ such that every graph has an edge-coloring with $\aleph_{1}$ colors such that all induced copies of $X$ get all colors.

THEOREM. It is consistent that there exists a graph $X$ on $\omega_{1}$ such that for every graph $Y$ there is a coloring of the edges of $Y$ with $\aleph_{1}$ colors with the property that every induced copy of $X$ in $Y$ gets every color.

P r o o f. Let $(P, \leq)$ be the following notion of forcing: $p \in P$ iff $p=(s, g)$ where $s \in\left[\omega_{1}\right]^{<\omega}, g \subseteq[s]^{2} ;\left(s^{\prime}, g^{\prime}\right) \leq(s, g)$ iff $s^{\prime} \supseteq s$ and $g=g^{\prime} \cap[s]^{2}$. If $G \subseteq P$ is a generic subset then $X=\bigcup\{g:(s, g) \in G\}$ is a graph on $\omega_{1}$. We show that in $V^{P}, X$ witnesses the truth of the Theorem.

To investigate a property of this forcing we work in $V[G]$. Assume that $\varphi$ is a sentence of the forcing language, $(s, g) \in G$ and some $e \in g$ has the following property: $(s, g) \Vdash \varphi$ and $(s, g-\{e\}) \Vdash \neg \varphi$. In this case we say that $e$ is critical for $\varphi$.

Claim. If $(s, g) \in G$ forces $\varphi$ then every edge critical for $\varphi$ is in $g$.

Proof. Assume that $\left(s^{\prime}, g^{\prime}\right) \in G$ shows that $e$ is critical for $\varphi$. Then $(s, g)$ and $\left(s^{\prime}, g^{\prime}\right)$ are compatible as both are in $G$ and $(s, g)$ and $\left(s^{\prime}, g^{\prime}-\{e\}\right)$ are incompatible as they force $\varphi, \neg \varphi$, respectively. This is only possible if $e \subseteq s \cap s^{\prime}$, i.e., $e \in g$.

In order to show that the statement of the Theorem holds in $V^{P}$ let $i:\left[\omega_{1}\right]^{2} \rightarrow \omega$ be a function with the property that $i(\beta, \alpha) \neq i\left(\beta^{\prime}, \alpha\right)$ for $\beta<\beta^{\prime}<\alpha$. Let $h:\left[\omega_{1}\right]^{2} \rightarrow \omega_{1}$ be a Todorčević function, i.e., $h^{\text {" }}[X]^{2}=\omega_{1}$ for every $X \in\left[\omega_{1}\right]^{\omega_{1}}$.

Assume that $1 \Vdash(\lambda, Y)$ is a graph. Assume, moreover, that $G \subseteq P$ is generic and $V[G]=\{\xi, \zeta\} \in Y$ and there is an edge $e$ critical to the statement $\{\xi, \zeta\} \in Y$ such that $i(e)>i(f)$ for every other edge $f$ critical to this statement. In this case we put $F(\xi, \zeta)=h(e)$. (We notice that this definition may leave some edges uncolored but this does not affect the result by the nature of the problem.) We show that this coloring of the edges of $Y$ satisfies the requirements.

Assume that $p \Vdash f: \omega_{1} \rightarrow \lambda$ embeds $X$, and an ordinal $\tau<\omega_{1}$ is given. For $\alpha \in \omega_{1}$ select $p_{\alpha} \leq p$ with $p_{\alpha} \Vdash f(\alpha)=\gamma_{\alpha}$. With the delta-system lemma for an uncountable $X \subseteq \omega_{1}$ the following hold: $p_{\alpha}=\left(s \cup s_{\alpha}, g_{\alpha}\right)$, $\alpha \in s_{\alpha}, g=g_{\alpha} \cap[s]^{2}$, so these conditions are compatible. With a further thinning we can assume that there is a number $n<\omega$ with $i(x, y) \leq n$ for 
$(x, y) \in g_{\alpha}$. For every $\alpha \in X$ the set $\{\beta \in X: i(\beta, \alpha) \leq n\}$ is finite so by the Hajnal set mapping theorem we can assume that in fact $i(\beta, \alpha)>n$ holds for every $\beta, \alpha \in X$. (A special case of that theorem says that if a finite set is associated with every countable ordinal then there is an uncountable subset of $\omega_{1}$ with ordinals not associated with each other. See Chapter 44 of [1].)

If we now put $p=\left(s \cup s_{\alpha} \cup s_{\beta}, g_{\alpha} \cup g_{\beta} \cup\{\alpha, \beta\}\right)$ for some $\beta \neq \alpha$ in $X$ with $h(\beta, \alpha)=\tau$ then $\{\beta, \alpha\}$ is critical to the statement $\left\{\gamma_{\alpha}, \gamma_{\beta}\right\} \in Y, p$ forces this, $i(\alpha, \beta)>i(e)$ for every $e \in g_{\alpha} \cup g_{\beta}$ so by the Claim and the definition of the coloring $p$ forces that $F\left(\gamma_{\alpha}, \gamma_{\beta}\right)=\tau$ and we are done.

\section{References}

[1] P. Erdős, A. Hajnal, A. Máté and R. Rado, Combinatorial Set Theory: Partition Relation for Cardinals, North-Holland, 1984.

[2] A. Hajnal and P. Komjáth, Embedding graphs into colored graphs, Trans. Amer. Math. Soc. 307 (1988), 395-409; corrigendum: 332 (1992), 475.

[3] S. Shelah, Consistency of positive partition theorems for graphs and models, in: Set Theory and Applications, J. Steprāns and S. Watson (eds.), Lecture Notes in Math. 1401, Springer, 1989, 167-193.

[4] S. Todorčević, Coloring pairs of countable ordinals, Acta Math. 159 (1987), 261294.

Department of Computer Science

Eötvös University

Múzeum krt. 6-8

1088 Budapest, Hungary

E-mail: kope@cs.elte.hu

Received 14 August 1996;

in revised form 3 November 1996 